\title{
Blockade of IL-23 ameliorates allergic lung inflammation via decreasing the infiltration of Tc17 cells
}

Sheng Cheng, Huilong Chen, Aili Wang, Hansvin Bunjhoo, Yong Cao, Jungang Xie, Yongjian Xu, Weining Xiong

Key Laboratory of Pulmonary Diseases of Health Ministry, Tongji Medical College, Huazhong University of Science and Technology, Wuhan, China

Submitted: 16 October 2014

Accepted: 14 January 2015

Arch Med Sci 2016; 12, 6: 1362-1369

DOI: $10.5114 /$ aoms.2016.62923

Copyright $\odot 2016$ Termedia \& Banach

\section{Abstract}

Introduction: Tc17 cells are interleukin (IL)-17-producing CD8 ${ }^{+} \mathrm{T}$ cells and have been found to participate in the development of allergic asthma. Interleukin-23 is a cytokine that may be involved in modulating the IL-17 response via Th17 cells. This study aimed to investigate whether IL-23 also has immunomodulatory effects on Tc17 cells.

Material and methods: An allergic asthmatic mouse model was induced by sensitizing and challenging with ovalbumin (OVA). Anti-IL-23 antibody was administered intratracheally before challenge to the OVA-induced asthmatic mouse model. Airway hyperresponsiveness, lung inflammation, Tc17 cell percentages and IL-17 level in the lung tissue homogenate were measured. Results: Anti-IL-23 treatment reduced airway hyperresponsiveness (Rn 2.471 \pm 0.5077 vs. $4.051 \pm 0.2334, p<0.05$ ), inflammatory cell infiltration in bronchoalveolar lavage fluid (eosinophils $140.0 \pm 9.869$ vs. $222.4 \pm 31.55, p<0.05$, neutrophils $75.93 \pm 6.745$ vs. $127.4 \pm 19.73, p<0.05$ ), airway inflammation and mucus secretion. Treatment with anti-IL-23 antibody also markedly reduced IL-17 level (398.1 \pm 28.74 vs. $590.6 \pm 36.13, p<0.01$ ) and percentage of Th17 and Tc17 cells in lung tissue homogenate ( $4.200 \pm 0.1581$ vs. $9.314 \pm 1.027, p<0.01$ and 2.852 \pm 0.2566 vs. $5.588 \pm 0.3631, p<0.01$, Th17 and Tc17 cells respectively).

Conclusions: Our data suggest that the IL-23/Tc17 cell axis may be involved in the pathogenesis of asthma as the complement of IL-23/Th17 cells.

Key words: asthma, interleukin-23, Tc17 cells, Th17 cells.

\author{
Corresponding author: \\ Weining Xiong \\ Key Laboratory of \\ Pulmonary Diseases \\ of Health Ministry \\ Tongji Medical College \\ Huazhong University \\ of Science and Technology \\ 1095 Jiefang Dadao Road \\ 430030 Wuhan, China \\ Phone: +862783663617 \\ E-mail: xiongdoctor@hotmail. \\ com
}

\section{Introduction}

Asthma is clinically characterized by reversible airflow obstruction, mucus hypersecretion and airway remodeling associated with inflammation [1]. Despite the effective therapies such as corticosteroids and other new therapeutics, about half of patients with asthma are poorly controlled [2]. The strongest risk factors for developing asthma are a combination of genetic predisposition with environmental exposure to inhaled substances and particles that may provoke allergic reactions. After allergen exposure, airway inflammation is orchestrated mainly by allergen-specific $T$ helper (Th) 2 and other T cells, which are recruited and accumulate in the lungs and produce a range of different effector cytokines, including interleukin (IL)-4, IL-5, IL-13 and tumor necrosis factor (TNF)- $\alpha$ [3]. In addition to Th2 cells, the role of IL-17-producing CD4 ${ }^{+} \mathrm{T}$ cells, named Th17 cells, in allergic diseases has lately become a subject of great interest. An increased level 
of IL-17 has been reported in lungs of patients with severe asthma [4]. Studies also show that Th17 cells may participate in the pathogenesis of coronary heart disease and myocardial infarction by affecting atherosclerosis [5]. Interleukin-23, a heterodimeric cytokine consisting of a p19 subunit specific for IL-23 and a p40 subunit shared with IL-12, has been shown to play an important role in the maintenance [6] and acquisition of pathogenic function of Th17 cells [7]. Indeed, the IL-23/ Th17 cell axis has been found to be associated with the development of various inflammatory diseases, such as arthritis [8], psoriasis [9], inflammatory bowel disease (IBD) [10] and asthma [11].

Besides Th17 cells, other CD8 ${ }^{+}$subtype T cells, named Tc17 cells, also produce IL-17. Tc17 cells are involved in human pathologies including primary biliary cirrhosis, immune thrombocytosis, psoriasis and cancer [12-15]. Our previous study and other studies demonstrated that Tc17 cells might be involved in the pathogenesis of allergic asthma [16-18].

In this context, the main goal of the study was to further investigate the immunomodulatory effects of IL-23 on Tc17 cells in a mouse model of asthma, through the administration of anti-IL-23 antibody.

\section{Material and methods}

\section{Mice}

Female BALB/c mice (6-8 weeks old) were purchased from the Hubei Laboratory Animal Center (Wuhan, China) and housed under specific pathogen-free conditions in the Laboratory Animal Center of Huazhong University of Science and Technology. The experimental procedure was approved by the committee of Huazhong University of Science and Technology for animal research.
Induction of airway allergic inflammation and administration of anti-mouse IL-23 neutralization antibody or isotype control

The asthmatic model was constructed as previously described [19]. Mice were divided into four groups as indicated, and each group was named according to sensitization/challenge/treatment: 1) sham group, sham/PBS/vehicle (normal control animals); 2) ovalbumin (OVA) group, OVA/OVA/vehicle (allergic control animals); 3) anti-IL-23 group, OVA/OVA/anti-IL-23 (allergic mice treated with anti-mouse IL-23 p19 neutralization antibody); and 4) isotype group, OVA/OVA/isotype Ab (allergic mice treated with isotype antibody, rat $\operatorname{lgG} 1$ ). In the indicated groups mice were administered anti-mouse IL-23 p19 neutralization antibody or isotype control (eBioscience, San Diego, CA) by intratracheal injection $1 \mathrm{~h}$ before the challenge, as previously reported [20]. The protocol is shown in Figure 1.

\section{Determination of airway hyperresponsiveness (AHR)}

Peak pulmonary resistance $\left(\mathrm{cm} \mathrm{H}_{2} \mathrm{O} \cdot \mathrm{ml}^{-1} \cdot \mathrm{s}^{-1}\right)$ was determined for AHR using the FlexiVent system $(10 \mathrm{ml} / \mathrm{kg}$ tidal volume at $150 \mathrm{breaths} / \mathrm{min}$, SCIREQ, Montreal, Quebec, Canada) after delivery of aerosolized methacholine (MCh) (Sigma, MO, USA). Methacholine aerosol (3.0-50 $\mathrm{mg} / \mathrm{ml}$ ) was administered for $10 \mathrm{~s}$, then airway resistance was continuously monitored and recorded.

\section{Bronchoalveolar lavage fluid analysis}

Bronchoalveolar lavage fluid (BALF) samples were obtained as described previously [21]. The lung of the mouse was flushed via a trachea cannula with $0.8 \mathrm{ml}$ of phosphate buffer saline (PBS) three times after the last OVA challenge. The la-

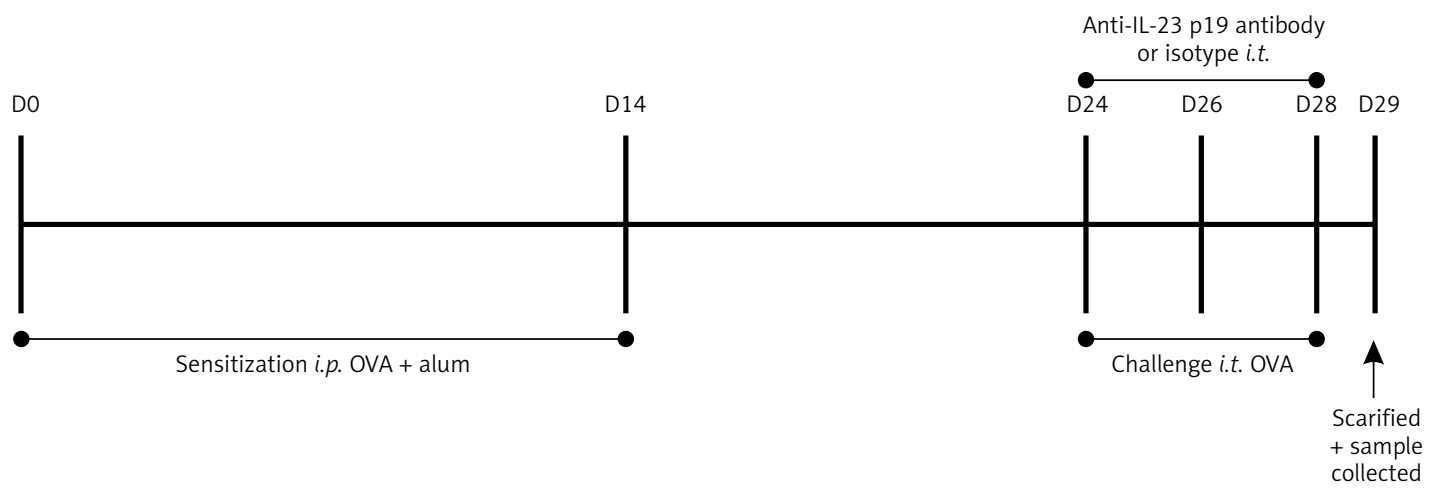

Figure 1. The experimental protocol. The ovalbumin (OVA) mice were sensitized on day 0 by i.p. injection of $20 \mu \mathrm{g}$ of OVA conjugated with $100 \mu \mathrm{l}$ of aluminum hydroxide. On day 14, mice were sensitized a second time with $100 \mu \mathrm{g}$ of OVA. On days 24,26 , and 28 , mice were anesthetized and challenged with $200 \mu \mathrm{g}$ of OVA in $40 \mu \mathrm{l}$ of sterile PBS by intratracheal instillation. The anti-IL-23 and isotype group mice were treated by intratracheal instillation of anti-IL-23 p19 Ab or rat IgG1 (isotype antibody for anti-IL-23 p19) $1 \mathrm{~h}$ before OVA challenge on days 24, 26, and 28 , respectively. The normal control mice received sterile PBS with aluminum hydroxide i.p. on days 0 and 14 and $40 \mu \mathrm{l}$ of sterile PBS on days 24,26 , and 28 by intratracheal instillation 
vage fluid was centrifuged at $200 \mathrm{~g}$ for $5 \mathrm{~min}$. The cell pellets were resuspended in $0.5 \mathrm{ml}$ of PBS and used for total and differential cell counts. The total number of cells in the BALF was counted by a hemacytometer. Differential cell counts were carried out after Diff-Quick staining.

\section{Lung histopathologic examination}

The left lung lobes were filled with $4 \%$ paraformaldehyde and embedded in paraffin. Five$\mu \mathrm{m}$ thick longitudinal sections from the left lobes were examined for airway inflammation and mucus secretion with hematoxylin and eosin (H \& E) and periodic acid-Schiff (PAS) stain, respectively, as described previously [22, 23].

\section{Lung homogenate preparation and IL-17 measurement}

The lung tissue suspensions were prepared as reported previously [24]. In brief, the right lung was dissected, then quickly frozen in liquid nitrogen before being stored at $-80^{\circ} \mathrm{C}$. After thawing, the lung tissue was homogenized in PBS and centrifuged at $3000 \mathrm{rpm}$ for $15 \mathrm{~min}\left(4^{\circ} \mathrm{C}\right)$ to remove sediments, then the supernatant was kept for cytokine measurements. The levels of IL-17 in lung tissue homogenate were determined by ELISA kits (Neobioscience, Beijing, China) in accordance with the manufacturer's instructions.

\section{Flow cytometric analysis of IL-17 expression of $\mathrm{T}$ cells in lung tissue}

A lung tissue single cell suspension was prepared as previously [11]. The cells from lung tissues were then stimulated by phorbol myristate acetate $(50 \mathrm{ng} / \mathrm{ml})$ (Sigma, MO, USA) and ionomycin (1000 ng/ml) (Sigma, MO, USA) for $5 \mathrm{~h}$.

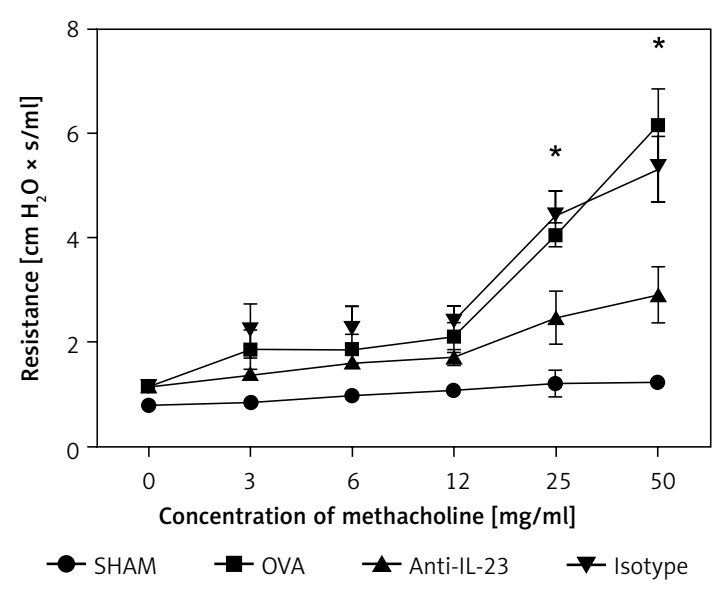

Figure 2. Alleviation of AHR by anti-IL-23 Ab. Airway resistance significantly increased after exposure to different concentrations of methacholine in OVA-challenged mice. Anti-IL-23 Ab alleviated the AHR significantly
Brefeldin A (50 ng/ml) (Sigma, MO, USA) was used to block the flow of cytokines from cytoplasm. Then, the cells were incubated with the appropriate concentration of PE-CY5 conjugated anti-CD3 mAb (Biolegend, San Diego, CA) and FITC-conjugated anti-CD8 mAb (Biolegend, San Diego, CA) for $30 \mathrm{~min}$ at $4^{\circ} \mathrm{C}$ in darkness. Then the cells were fixed and permeabilized with $\mathrm{Cy}$ tofix/Cytoperm solution (BD Biosciences, San Jose, CA) for intracellular cytokine staining. After incubation with PE conjugated anti-IL-17A mAb (Biolegend, San Diego, CA) for $30 \mathrm{~min}$ at $4^{\circ} \mathrm{C}$ in darkness, flow cytometry was performed on a BD LSR II cytometer to determine the percentages of Tc17 (CD3 $\left.{ }^{+} \mathrm{CD}^{+}{ }^{+} \mathrm{LL}-17^{+}\right)$or Th17 $\left(\mathrm{CD}^{+} \mathrm{CD} 8 \mathrm{IL}-17^{+}\right)$ lymphocytes.

\section{Statistical analysis}

All data are presented as means \pm SEM. One-way analysis of variance (ANOVA) was applied to evaluate statistical significance. $P<0.05$ was considered to be significant. Graph generation and statistical analysis were performed with GraphPad Prism5.

\section{Results}

\section{Alleviation of AHR by anti-IL-23 Ab administration}

We evaluated whether the blockade of IL-23 contributed to a change in airway mechanics after exposure to the bronchoconstricting agent MCh. We used a computer-controlled small animal ventilator (flexiVent) to compare changes in Rn. Exposure to MCh resulted in a significant increase of

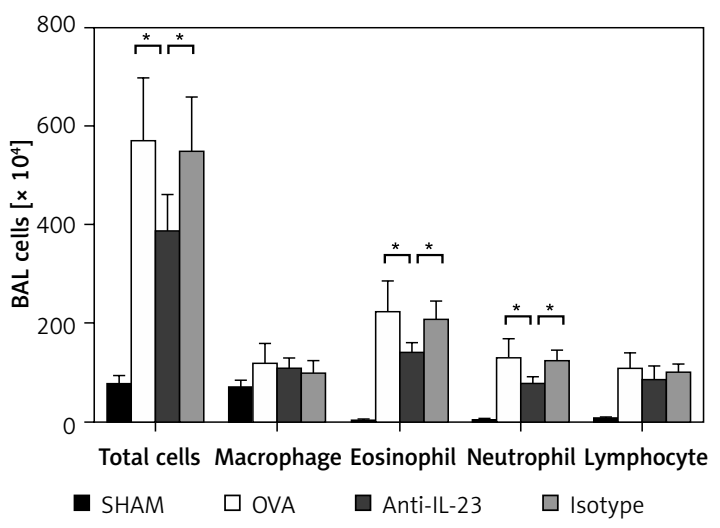

Figure 3. Anti-IL-23 Ab inhibited inflammatory cell infiltration in BALF. Total cells and macrophages, neutrophils, eosinophils and lymphocytes were significantly increased in BALF of OVA and isotype group mice compared with control mice. AntiIL-23 Ab administration significantly decreased eosinophils and neutrophils in BALF and slightly decreased lymphocytes compared with OVA-sensitized and OVA-challenged mice

Data were plotted as means \pm SEM of two independent experiments. ${ }^{*} P<0.05$. 
$\mathrm{Rn}$ in the OVA-challenged mice. This up-regulation of $\mathrm{Rn}$ in OVA-challenged mice was effectively reduced by blockade of IL-23 (Figure 2).

\section{Inhibition of inflammatory cell infiltration in BALF by anti-IL-23 Ab}

The numbers of total cells and macrophages, neutrophils, eosinophils, and lymphocytes were significantly increased in BALF of OVA group mice compared with control mice. Anti-IL-23 Ab administration significantly decreased numbers of eo-
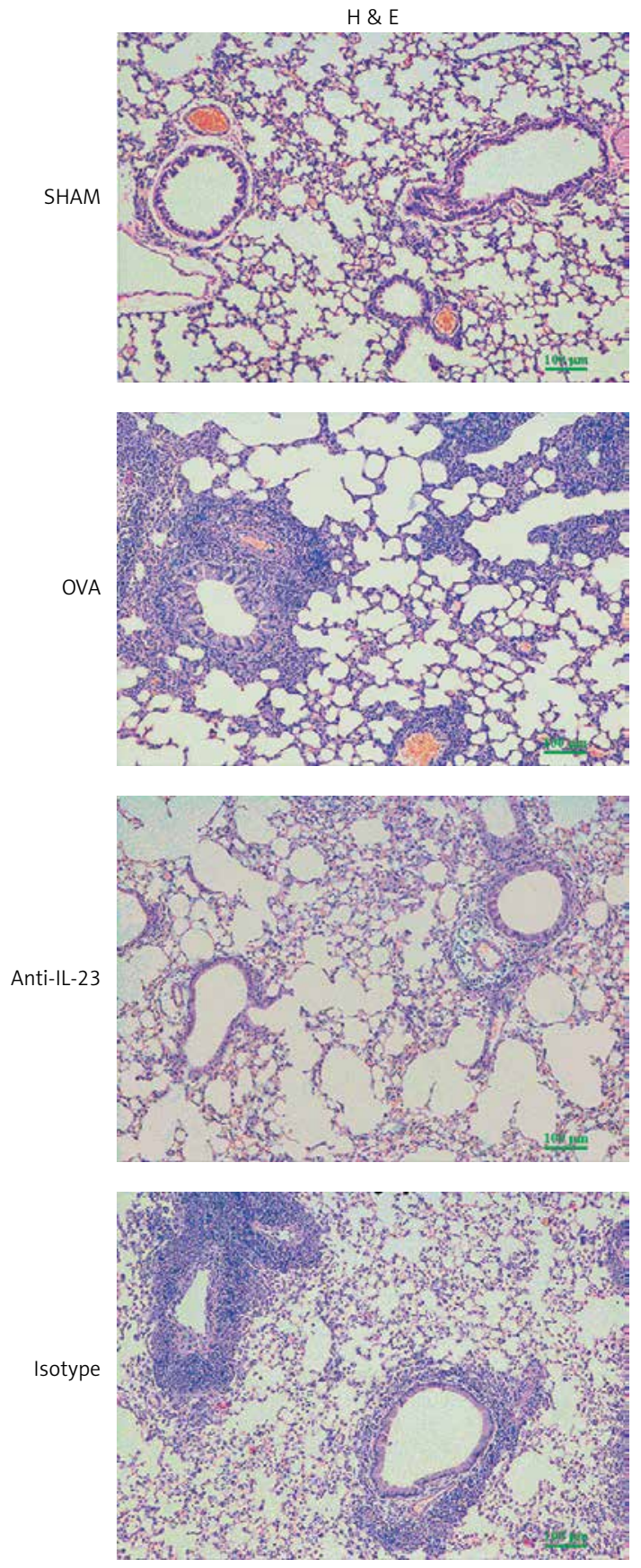

Figure 4. Anti-IL-23 Ab attenuates airway inflammation and mucus hypersecretion in asthmatic mice. Lung sections stained with hematoxylin and eosin (H \& E, 100x) and periodic acid-Schiff (PAS, 200x). Anti-IL-23 Ab reduced the accumulation of inflammatory cells in the peribronchial and perivascular regions, as well as the mucus secretion sinophils and neutrophils in BALF compared with OVA-challenged mice (Figure 3).

Intratracheal delivery of anti-IL-23 Ab alleviated allergic inflammation and mucus hypersecretion

In the OVA group, inflammatory cells accumulated around the bronchus and vessels after OVA challenge. Administration of anti-IL-23 Ab reduced the accumulation of inflammatory cells in the peribronchial and perivascular regions. Asthmat-
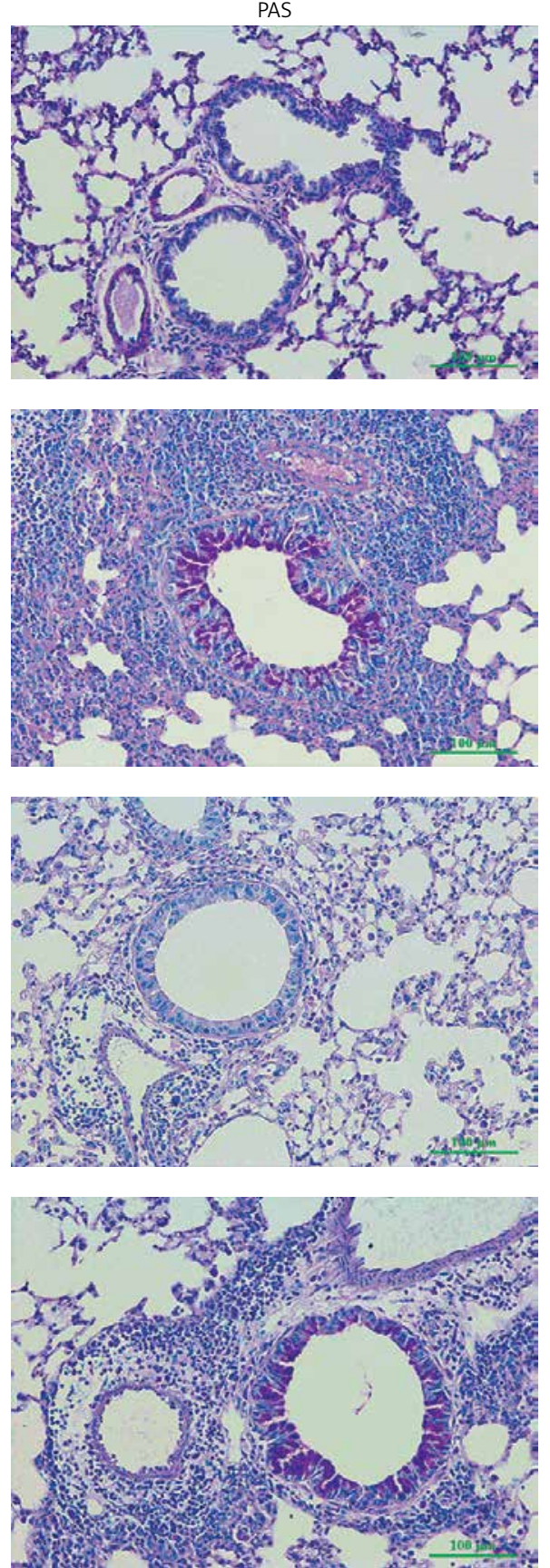


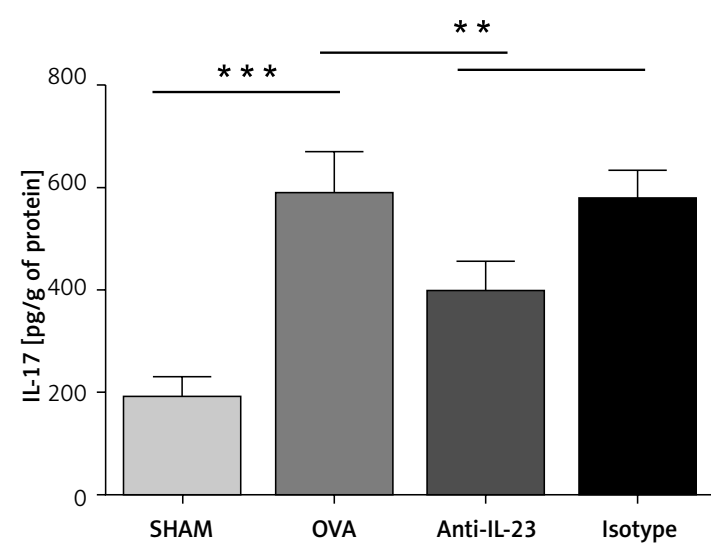

Figure 5. Anti-IL-23 Ab reduced IL-17 level in mice with allergic airway inflammation. The IL-17 levels in lung tissue homogenate were determined by ELISA. The IL-17 level was up-regulated in OVA-challenged mice and anti-IL-23 Ab reduced the up-regulation significantly

Data are expressed as the mean \pm SEM $(n=6$ in each group). Results are representative of two independent experiments with similar results; ${ }^{* *} p<0.01,{ }^{* * *} p<0.001$.

ic mice also showed increased mucus secretion in PAS staining compared with that seen in normal control mice, and anti-IL-23 Ab inhibited this mucus secretion. These effects were not observed in the isotype antibody treated group (Figure 4).

\section{Intratracheal delivery of anti-IL-23 Ab reduced IL-17 level in mice with allergic airway inflammation}

The IL-17 level in lung tissue homogenate was up-regulated in OVA-challenged mice compared with control mice (OVA vs. SHAM: $590.6 \pm 36.13$ $\mathrm{pg} / \mathrm{g}$ vs. $192.2 \pm 18.60 \mathrm{pg} / \mathrm{g})$. This up-regulation was significantly reduced by anti-IL-23 Ab intratracheal delivery (anti-IL-23 vs. OVA: $398.1 \pm 28.74$ $\mathrm{pg} / \mathrm{g}$ vs. $590.6 \pm 36.13 \mathrm{pg} / \mathrm{g}$ ), whereas no reduction was observed with isotype antibody (Figure 5).

\section{Decreased Tc17 and Th17 cell infiltration in lung by blockade of IL-23}

To determine whether the effect of anti-IL-23 $\mathrm{Ab}$ was achieved through restraining the infiltration of IL-17 producing T cells, especially Tc17 cells into the lungs in asthma, we investigated Tc17 cells and Th17 cell infiltration by flow cytometry. As shown in Figure 6, not only Th17 cells but also Tc17 cell infiltration could be inhibited by blockade of IL-23, though the flow cytometry dot plots seem not too much different when seen with the naked eye due to the smaller absolute numbers of Tc17 cells. The number of inflammatory cells from a single sham-treated mouse (normal control mouse) was too small to perform flow cytometry analysis, so we had to combine three sham-treated mice to do it; then sham-treated an- imals showed a higher inflammatory cell number in the lungs.

\section{Discussion}

The role of IL-17 in asthma was proven. Bullens et al. demonstrated that the level of IL-17A mRNA was elevated in the sputum sample of asthmatic patients [25]. An increased serum IL-17 level was reported in asthma, which might be one of the risk factors of severe asthma [26]. In a mouse model, allergic sensitization through the airways or the skin promoted bronchial IL-17 responses and induced AHR, while deficiency in IL-17 led to an impaired response to allergens and lack of AHR [27, 28]. In our study we also found elevated IL-17 level in lung tissue and AHR in the OVA-induced asthmatic group, which was consistent with previous studies.

Airway mucus hypersecretion narrows the airway lumen and limits airflow, and thus contributes to airway remodeling. Regulating the development of Th1/Th2 subsets could inhibit airway remodeling in asthmatic mice in other research [29]. Evidence has also shown the relationship of IL-17 and airway mucus secretion. Chen et al. reported that recombinant IL-17A upregulated the mucin gene expression of MUC5AC and MUC5B in primary human tracheobronchial epithelial cells [30]. In mice, respiratory syncytial virus (RSV) infection during ongoing allergic airway inflammation increased mucous cell metaplasia and mucin protein expression, and IL-17A was significantly increased in whole-lung homogenates [31]. In our study, increased mucus secretion was also found in OVA-induced allergic mice accompanied by increasing IL-17 level in lung tissue, supporting those previous studies.

Previous studies have also shown the involvement of IL-23 in asthma. Ciprandi et al. found that serum IL-23 levels were increased in allergic asthmatic children not treated with corticosteroids, and IL-23 levels were strongly and inversely correlated with lung function [32]. In antigen-sensitized mice, a study demonstrated that IL-23 p19 mRNA was up-regulated in the lung [33]. Furthermore, the proinflammatory features of IL-23 have been linked with Th17 cell responses, through expansion and maintenance of the Th17 cells [34]. The polarization of Th17 cells was mainly regulated by the key polarized cytokines IL-23 and IL-6 [35]. Recently, studies have shown that IL-23-IL-23R signaling and the IL-23/ Th17 cell axis promote allergic airway inflammation $[11,36]$.

However, Tc17 cells, a subgroup of CD8 $8^{+} \mathrm{T}$ lymphocytes, also produce IL-17. Previous studies had reported that Tc17 cell differentiation was closer to that described for Th17 cells, including the expression of cytokines such as IL-17 and IL-21, the 
A

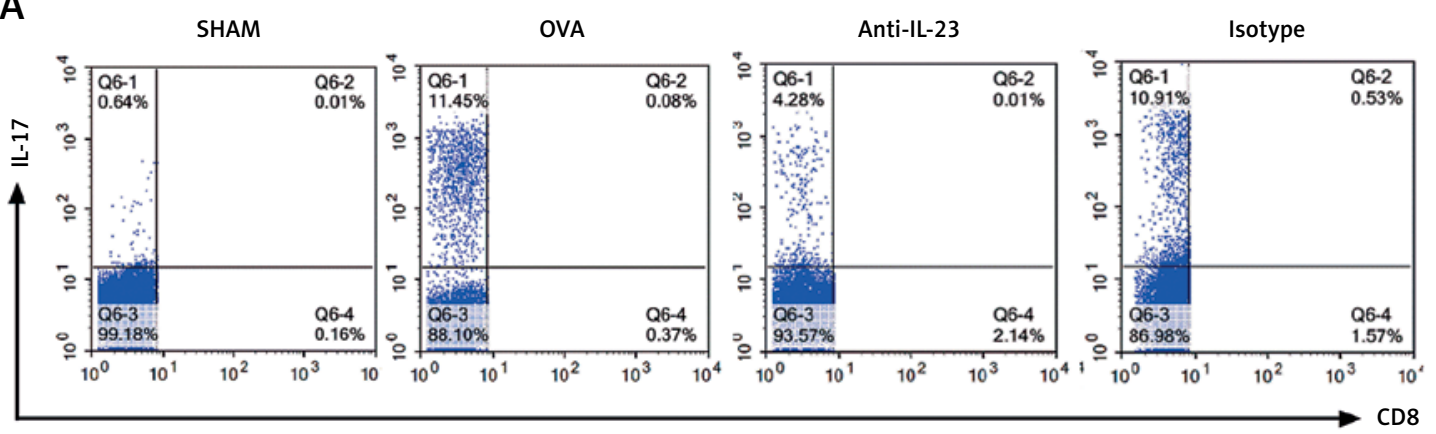

B

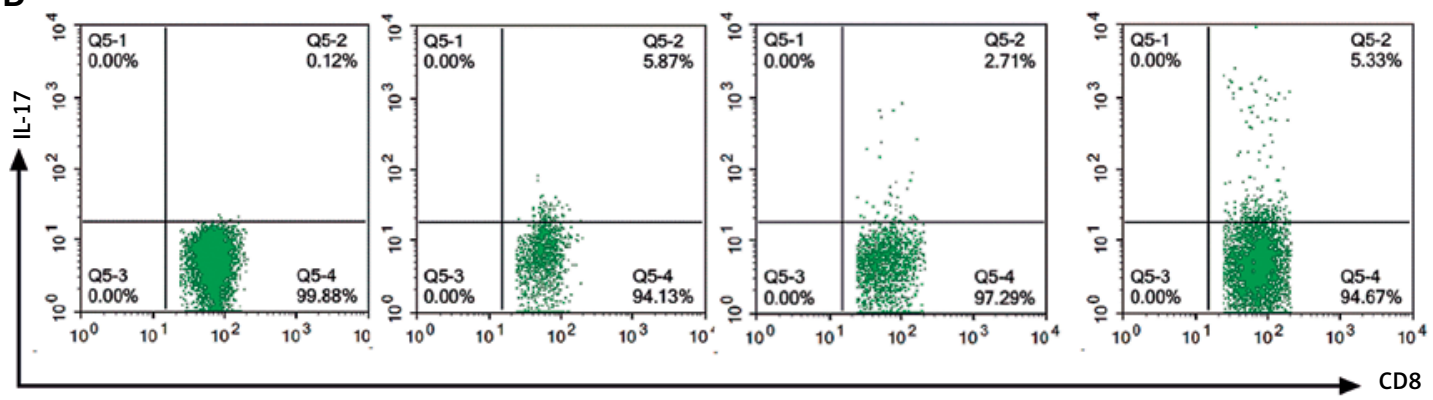

C

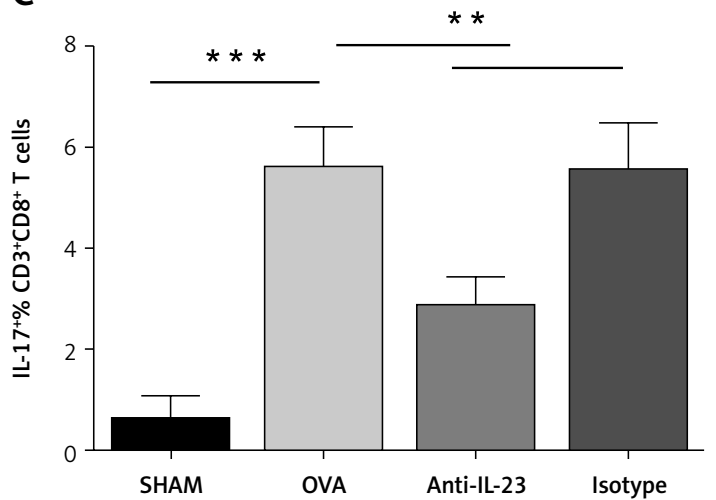

D

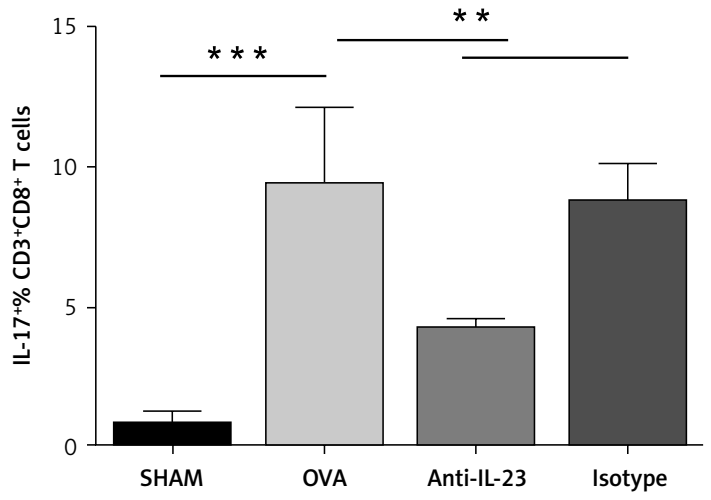

Figure 6. Decreased TC17 and Th17 cell infiltration in lung by blockade of IL-23. A-B - Representative flow cytometry dot plots for each treatment group. All plots were gated on the lymphocyte population based on $\mathrm{CD}^{+} \mathrm{CD} 8^{-}(\mathrm{A})$ and $\mathrm{CD}^{+} \mathrm{CD}^{+}$(B). Numbers in the upper left (A) or upper right (B) quadrant indicate the percentage of $\mathrm{IL}-17^{+}$ $T$ cells among the indicated lymphocyte population. $C$ - Percentages of $I L-17^{+} C D 3^{+} C D 8^{-} T$ cells for each treatment group. D - Percentages of $\mathrm{IL}-17^{+} \mathrm{CD} 3{ }^{+} \mathrm{CD} 8{ }^{+} \mathrm{T}$ cells for each treatment group

Data are expressed as the mean \pm SEM ( $n=6$ in each group). Results are representative of two independent experiments with similar results; ${ }^{* *} p<0.01,{ }^{* * *} p<0.001$

IL-23 receptor and the lineage-specific transcription factors ROR $\alpha$ and ROR $\gamma$ t [37]. Our previous study showed that the percentages of Th17 cells and Tc17 cells were higher in both patients with asthma and asthmatic mice compared with controls [16]. Other studies also indicated the involvement of Tc17 cells in the pathogenesis of asthma $[17,18]$. The goal of this study therefore was to explore whether the IL-23/Tc17 cell axis also exists in an OVA-induced asthmatic mouse model.

In the OVA-induced group, Tc17 cells and Th17 cells were up-regulated in lung tissue compared with negative control subjects, accompanied by an elevated IL-17 level in lung tissue homogenate.
Anti-IL-23 Ab treatment reduced Tc17 cell and Th17 cell infiltration and the IL-17 level, and subsequently significantly alleviated airway inflammation, as well as eosinophil and neutrophil infiltration in BALF, mucus hypersecretion, and OVA-induced AHR. In our experiment, the numbers of lymphocytes were not reduced significantly by anti-IL-23 treatment. We think that there might be two reasons. First, although IL-23 stimulates differentiation of naïve T-lymphocytes into Th/Tc17 cells, the total number of T-lymphocytes may not change during the process; second, besides the Th/Tc17 cells there are other subtypes of T-lymphocytes in the pathogenesis of asthma, and their differentiation may not be 
influenced by IL-23. So this may lead to no significant change in the number of lymphocytes.

Tc17 cells were also one kind of effector T cell subset involved in the induction of inflammation and autoimmune tissue injury [38, 39]. Recently, studies have suggested that Tc17 cooperates with Th17 in similar functions in inflammatory conditions, including autoimmune diseases, infection, and antitumor immunity [38, 40]. Our findings further demonstrated that the cell sources of IL-17 in allergic lung inflammation were not only $\mathrm{CD}^{+}{ }^{+} \mathrm{T}$ cells but also $\mathrm{CD} 8^{+} \mathrm{T}$ cells, and Tc17 cells and Th17 cells may both be involved in the mechanism of asthma.

In conclusion, our findings demonstrated not only the IL-23/Th17 cell axis but also the IL-23/ Tc17 cell axis in allergic airway inflammation. The role of Tc17 cells in allergic diseases needs to be investigated more deeply.

\section{Acknowledgments}

This work was supported by the National Natural Science Foundation of China (No. 81170021 and No. 30900647).

\section{Conflict of interest}

The authors declare no conflict of interest.

\section{References}

1. Barnes PJ. Immunology of asthma and chronic obstructive pulmonary disease. Nat Rev Immunol 2008; 8: 183-92.

2. Marandi Y, Farahi N, Hashjin GS. Asthma: beyond corticosteroid treatment. Arch Med Sci 2013; 9: 521-6.

3. Galli SJ, Tsai M, Piliponsky AM. The development of allergic inflammation. Nature 2008; 454: 445-54.

4. Al-Ramli W, Préfontaine D, Chouiali F, et al. Th17-associated cytokines (IL-17A and IL-17F) in severe asthma. J Allergy Clin Immunol 2009; 123: 1185-7.

5. Chalubinski M, Wojdan K, Dorantowicz R, et al. Comprehensive insight into immune regulatory mechanisms and vascular wall determinants of atherogenesis - emerging perspectives of immunomodulation. Arch Med Sci 2013; 9: 159-65.

6. Langrish CL, Chen Y, Blumenschein WM, et al. IL-23 drives a pathogenic $T$ cell population that induces autoimmune inflammation. J Exp Med 2005; 201: 233-40.

7. McGeachy MJ, Bak-Jensen KS, Chen Y, et al. TGF-beta and IL- 6 drive the production of IL-17 and IL-10 by T cells and restrain Th-17 cell-mediated pathology. Nat Immunol 2007; 8: 1390-7,

8. Cornelissen F, van Hamburg J P, Lubberts E. The IL-12/ IL-23 axis and its role in Th17 cell development, pathology and plasticity in arthritis. Curr Opin Investig Drugs 2009; 10: 452-62.

9. Di Cesare A, Di Meglio P, Nestle FO. The IL-23/Th17 axis in the immunopathogenesis of psoriasis. J Invest Dermatol 2009; 129: 1339-50.

10. Sarra M, Pallone F, MacDonald TT, Monteleone G. IL-23/ IL-17 axis in IBD. Inflamm Bowel Dis 2010; 16: 1808-13.

11. Wakashin H, Hirose K, Maezawa Y, et al. IL-23 and Th17 cells enhance Th2-cell-mediated eosinophilic airway in- flammation in mice. Am J Respir Crit Care Med 2008; 178: 1023-32.

12. Oo YH, Banz V, Kavanagh D, et al. CXCR3-dependent recruitment and CCR6-mediated positioning of Th- 17 cells in the inflamed liver. J Hepatol 2012; 57: 1044-51.

13. Hu Y, Ma DX, Shan NN, et al. Increased number of Tc17 and correlation with Th17 cells in patients with immune thrombocytopenia. PLoS One 2011; 6: e26522.

14. Res PC, Piskin G, de Boer OJ, et al. Overrepresentation of IL-17A and IL-22 producing CD8 T cells in lesional skin suggests their involvement in the pathogenesis of psoriasis. PLoS One 2010; 5: e14108.

15. Zhang Y, Hou F, Liu X, et al. Tc17 cells in patients with uterine cervical cancer. PLoS One 2014; 9: e86812.

16. Li K, Wang Z, Cao Y, et al. The study of the ratio and distribution of Th17 cells and Tc17 cells in asthmatic patients and the mouse model. Asian Pac J Allergy Immunol 2013; 31: 125-31.

17. Zhao Y, Yang J, Gao YD. Altered expressions of helper $T$ cell (Th)1, Th2, and Th17 cytokines in CD8(+) and gammadelta T cells in patients with allergic asthma. J Asthma 2011; 48: 429-36.

18. Reber LL, Daubeuf F, Plantinga M, et al. A dissociated glucocorticoid receptor modulator reduces airway hyperresponsiveness and inflammation in a mouse model of asthma. J Immunol 2012; 188: 3478-87.

19. Rangasamy T, Guo J, Mitzner WA, et al. Disruption of Nrf2 enhances susceptibility to severe airway inflammation and asthma in mice. J Exp Med 2005; 202: 47-59.

20. Penido C, Costa MF, Souza MC, et al. Involvement of CC chemokines in gammadelta $T$ lymphocyte trafficking during allergic inflammation: the role of CCL2/CCR2 pathway. Int Immunol 2008; 20: 129-39.

21. Nakagome K, Imamura M, Okada H, et al. Dopamine D1-like receptor antagonist attenuates Th17-mediated immune response and ovalbumin antigen-induced neutrophilic airway inflammation. J Immunol 2011; 186: 5975-82.

22. Agrawal A, Rengarajan S, Adler KB, et al. Inhibition of mucin secretion with MARCKS-related peptide improves airway obstruction in a mouse model of asthma. J Appl Physiol (1985) 2007; 102: 399-405.

23. Sun Y, Wang J, Li H, et al. The effects of budesonide on angiogenesis in a murine asthma model. Arch Med Sci 2013; 9: 361-7.

24. You QH, Zhang D, Niu CC, et al. Expression of IL-17A and IL-17F in lipopolysaccharide-induced acute lung injury and the counteraction of anisodamine or methylprednisolone. Cytokine 2014; 66: 78-86.

25. Bullens DM, Truyen E, Coteur L, et al. IL-17 mRNA in sputum of asthmatic patients: linking $T$ cell driven inflammation and granulocytic influx? Respir Res 2006; 7: 135.

26. Agache I, Ciobanu C, Agache C, Anghel M. Increased serum IL-17 is an independent risk factor for severe asthma. Respir Med 2010; 104: 1131-7.

27. Wilson RH, Whitehead GS, Nakano $\mathrm{H}$, et al. Allergic sensitization through the airway primes Th17-dependent neutrophilia and airway hyperresponsiveness. Am J Respir Crit Care Med 2009; 180: 720-30.

28. He R, Oyoshi MK, Jin H, Geha RS. Epicutaneous antigen exposure induces a Th17 response that drives airway inflammation after inhalation challenge. Proc Natl Acad Sci USA 2007; 104: 15817-22.

29. Yan L, Xiao-Ling S, Zheng-Yan C, et al. HSP70/CD80 DNA vaccine inhibits airway remodeling by regulating the transcription factors T-bet and GATA-3 in a murine model of chronic asthma. Arch Med Sci 2013; 9: 906-15. 
30. Chen $Y$, Thai P, Zhao $Y$ H, et al. Stimulation of airway mucin gene expression by interleukin (IL)-17 through IL-6 paracrine/autocrine loop. J Biol Chem 2003; 278: 17036-43.

31. Zhao J, Lloyd CM, Noble A. Th17 responses in chronic allergic airway inflammation abrogate regulatory T-cell-mediated tolerance and contribute to airway remodeling. Mucosal Immunol 2013; 6: 335-46.

32. Ciprandi G, Cuppari C, Salpietro AM, et al. Serum IL-23 strongly and inversely correlates with FEV1 in asthmatic children. Int Arch Allergy Immunol 2012; 159: 183-6.

33. Schnyder-Candrian S, Togbe D, Couillin I, et al. Interleukin-17 is a negative regulator of established allergic asthma. J Exp Med 2006; 203: 2715-25.

34. Langrish CL, Chen Y, Blumenschein WM, et al. IL-23 drives a pathogenic $T$ cell population that induces autoimmune inflammation. J Exp Med 2005; 201: 233-40.

35. Lewkowich IP, Lajoie S, Clark JR, et al. Allergen uptake, activation, and IL-23 production by pulmonary myeloid DCs drives airway hyperresponsiveness in asthma-susceptible mice. PLoS One 2008; 3: e3879.

36. Peng J, Yang XO, Chang SH, Yang J, Dong C. IL-23 signaling enhances Th2 polarization and regulates allergic airway inflammation. Cell Res 2010; 20: 62-71.

37. Huber M, Heink S, Grothe H, et al. A Th17-like developmental process leads to $\mathrm{CD} 8(+)$ Tc17 cells with reduced cytotoxic activity. Eur J Immunol 2009; 39: 1716-25.

38. Henriques A, Inês L, Couto $M$, et al. Frequency and functional activity of Th17, Tc17 and other T-cell subsets in systemic lupus erythematosus. Cell Immunol 2010; 264 97-103.

39. He D, Wu L, Kim HK, et al. CD8+ IL-17-producing T cells are important in effector functions for the elicitation of contact hypersensitivity responses. J Immunol 2006; 177: 6852-8.

40. Huber M, Heink S, Pagenstecher A, et al. IL-17A secretion by CD8+ T cells supports Th17-mediated autoimmune encephalomyelitis. J Clin Invest 2013; 123: 247-60. 